\title{
The World Building Framework for Immersive Storytelling Projects
}

\author{
Yan Breuleux ${ }^{1}$, Bruno de Coninck $^{1}$, Simon Therrien ${ }^{1}$ \\ ${ }^{1}$ Université du Québec à Chicoutimi, NAD-UQAC, 2566 Rue de Bleury, 7e étage Montréal (Québec) \\ H3A 2J2, Canada
}

\begin{abstract}
This article explores issues associated with immersive storytelling in order to examine how the field of World Building can constitute a theoretical framework for practice in the context of VR-based and Full Dome artistic projects. With respect to immersion, the intent will be to interpret the concept of storytelling in relation with the recent formulation of the concept of extended reality (XR). The very concept of World Building is transauthor and transmedia by nature. The transauthor dimension of World Building resides in the idea of subcreation, i.e., designing environments and interaction rules that help create a storytelling basis for generating multiple stories. Once the universe has been conceived, stories written by different authors take shape through transmedia processes across multiple distribution media (film, video games, web, etc.). The question then arises: How can the World Building approach shape the construction of immersive experiences? The article sets out to answer this question, and in doing so, to contribute to the research on environmental storytelling.
\end{abstract}

\section{Introduction}

This article explores issues associated with immersive storytelling in order to examine how World Building can constitute a theoretical framework for practice in the context of virtual reality and Full Dome artistic projects. Our articulation of this theoretical framework is inspired by the conceptions of researchers and practitioners from the film and video game domains. This explains why the diverse sources for this article include books, articles, specialized websites, presentation excerpts, and interviews available online. We respectively present the conceptions of several figureheads of the narratological current of game studies such as Mark J.P. Wolf, Henry Jenkins and Marie Laure Ryan. As for practitioners, we discuss three designers: 1- Alex McDowell, film production designer and researcher; 2- Monika Bielskyte, futurist \& future designer; and 3- Don Carson, game and theme park designer. These three profiles will allow for describing a particular conception of World 
Building and environmental storytelling that is focused on practice. After sketching a broad picture of Digital Storytelling, we explore the World Building concept in relation with the notion of environmental storytelling. This lens will then be used to examine research-creation projects.

The aim of this article is thus to provide an outline of a World Building practice for the field of Full Dome and virtual reality experience design. To do so, it is important to begin by establishing a field of research for teaching and transmission in the field of the creative industries (i.e., digital art, visual effects and video games). Next, we attempt to shed light on how immersive installations shape the mise en scène of narrative dimensions. After proposing a typology of immersive environments, we examine several research-creation projects carried out at the École des Arts Numériques de l'Animation et du Design / School of Digital Technology and Animation (NAD-UQAC) and at the Société des Arts Technologiques de Montréal / Montreal Society for Arts and Technology (SAT).

\section{Digital, Virtual, Interactive Storytelling}

Digital Storytelling, as a field of study, emerged at the crossroads of common interests in the research fields of digital art, literature, communication, and computer sciences in conjunction with the development of new media and entertainment industries. These industries include Massively Multiplayer Online Role-Playing Games (MMORPGs), interactive and immersive experiences of all kinds, smart toys, etc. [1] Given the vast array of applications and practices arising from this field, it should be pointed out that digital components and storytelling are cross-cutting concepts that allow for much crossover between disciplines. In light of the applications and installations produced by researchers in the field, Digital Storytelling embodies the promises of the confluence of the new digital economy and technoculture.

As a brief historical reminder, storytelling dimensions have always been central to the development of new apparatuses and installations. When patenting the Telesphere Mask on October 4, 1960, Morton Helig referred to a "Stereoscopic-television appartus for individual use" [2]. Hence, he was already establishing himself as articulating a new entertainment apparatus. Subsequently, in 1966, Ivan Sutherland created the first real-time 3D animation experience with his virtual reality headset, named the "Sword of Damocles"- a name that aptly conveyed how heavy the device was. In his article "The Ultimate Display," Sutherland interestingly mentions the metaphor of Alice in Wonderland [3]. Virtual reality indeed emerged as a technological embodiment of the famed journey through the looking-glass that plunges Alice into her fantastic world where anything is possible. By making this parallel with a literary classic, Sutherland concretely describes the fantastic nature of this new medium, which, without this metaphor, may have struck some as rather abstract and therefore harder to grasp. In doing so, he also successfully anchors this new medium in the timeline of history by assigning it a specific and significant role from the standpoint of the public. Both of these virtual reality apparatuses - mask and headset - anticipated, or rather concretely realized (but without today's powerful calculation capabilities) the fundamental configuration of modern virtual reality devices.

If we were to look for a pivotal moment for digital storytelling in terms of a diversification of practices and the active assertion of the term, it could be located from the beginning to the mid-1990s [4]. With the development, democratization of the computer and the multimedia web browser (the Mosaic browser in 1994), new digital media supports and 
advances in 3D simulation, a new form of interactivity gained ground, based on real-time connectivity to databases accessible locally and/or online. The utopian period of the 1990s was characterized by the hypothesis of the advent of a new language borne by digital technologies, particularly immersive ones. Janet Horowitz Murray, with his book Hamlet on the Hollodeck initially published in 1997, became a leading figure on this topic, and continues to be a key reference to this day [5]. The research in this field also includes the proceedings of a series of computer science conferences that started in 2001 on the overarching theme of Virtual Storytelling (ICVS). As mentioned in the introduction to the first conference in this series, "The first International Conference on Virtual Storytelling gathers researchers from the scientific, artistic, and industrial communities to demonstrate new methods and techniques, show the latest results, and to exchange concepts and ideas for the use of Virtual Reality technologies for creating, scripting, populating, rendering, and interacting with stories, whatever their form, be it theatre, movie, cartoon, advertisement, puppet show, multimedia work, video-games..." [6]. In 2008, the term "virtual" in the conference titles was replaced with "interactive." It is worth noting that Janet Horowitz Murray, whose works are often cited as a theoretical framework, was the keynote speaker for the 2018 conference [7]. The utopia of creating a $360^{\circ}$ literary remediation of a translation of Hamlet was realized in 2019 by the Commonwealth Shakespeare company in collaboration with Tech Giant Google [8].

Currently, to explore recent technological innovations in interactive storytelling, the community of practice associated with the Future of Storytelling (FoST) current has been taking an interest in the promotion of artists, theoreticians, researchers, entrepreneurs, designers, directors, etc., but also in research on new experiential practices [9]. Immersion issues hold a prominent place in this community. To emphasize the pluridisciplinarity of FoST contributions, it should be pointed out that this new field of creation also extends to documentary practices. For example, the laboratory of researcher William Uricchio, of the MIT Open Documentary Lab, explores, among other things, the experiential and informative potential of the virtual reality documentary [10] According to Émilie Ropert-Dupont, the practice of virtual journalism raises interesting questions about the role and purpose of emotions in transmitting information via immersive media [11]. Quebec is home to an exhibit series on the theme of Sensory Studies that partakes in the FoST current. This series is of cultural significance, given that it is presented at Montreal's Centre PHI every year and is affiliated with the founder of the Future of Storytelling, Charles Melcher [12]. The most recent conference in this series, entitled "The New Storytellers," featured Douglas Trumbull as its keynote speaker. The remarks made by Trumbull, who was responsible for the visual effects in the film 2001: A Space Odyssey (produced by Stanley Kubrick), [13] addressed the practice and language issues surrounding immersive cinema [14]. To clarify the subject of this article in relation with Trumbull's thinking, we are witnessing the advent of a new medium shaped by the viewer's special position in a situation of immersion. The first-person point of view participates in co-constructing the story of the immersive experience. Indeed, immersing the viewer in the story makes him or her a full-fledged player within it. Michael Gödde et al. describe this practice as "VR film." They also mention that producing a $360^{\circ}$ story requires guiding viewers' attention to the important elements in the scene, determining whether the "viewer" is an active participant or passive observer, choosing the right point of view for the immersive camera in order to determine the anchoring point in the environment, and thinking through the relationship between the temporality of the story, on one hand, and its relation to space, on the other. Finally, the concepts of framing and montage also need to be rethought in this context. The issue of the point of view and the language of immersion is also addressed in reflections by authors such as Celine Tricart and John Bucher [15]. 
These aspects show how the introduction of immersive cameras turns the cinematographic narrative structure on its head. In sum, the fundamental question of digital storytelling remains: How do digital technologies transform and shape the construction of the storytelling dimensions of the experience?

\subsection{Storytelling and technological environments}

The recent rise and proliferation of virtual technologies has led to the need to update the concept of virtual-reality continuum as proposed by Paul Milgram and his team in 1995 [16]. The concept of extended reality or cross reality (XR) has recently emerged in response to this need [17]. The concept is interesting to consider in that it allows for merging mixed reality technologies, including virtual reality, augmented reality and new forms of spatial virtualization emerging from ubiquitous computing. In this context, and with the increase in cell phones' recording, analysis and calculation capabilities, a hybridization of virtual and ubiquitous technologies may reasonably be expected in the (relative) long term. Without getting into the statistical details, it is very likely that virtual reality will be democratized primarily via the Internet of Things. This progression of technologies makes it possible to capture and model spaces and bodies in real time. For example, Google's previous smartphone research program Tango was able to project point cloudes in order to capture volumetric data in the user's environment. The program's spatial modelling allowed for designing augmented reality applications and games adapted to the particularities of the specific architectural space at hand. In addition, being able to digitally capture faces suggests the future possibility of adapting data to the specific profile of each individual. This means that our connected devices would transmit their actual real-world anchor point in space but also the topological data captured in each location. In turn, this would mean that any real-life environment could become the basis for a personalized immersive experience.

To better understand how such technologies shape storytelling dimensions, we have divided immersive environments into five types. The goal behind this typology is to define the conditions under which storytelling is developed and the extent to which it is possible to adapt and/or create a narrative and sensory experience. More specifically, we drew up our typology of immersive virtual environments (IVEs) following the logic of extended environments (XR). This typology is based on five forms of technological environments: 1-virtual reality environments, 2-mixed reality (MR) environments, 3-ubiquitous environments, 4- recorded environments, and 5-projected environments (outside the computers). Given that virtual reality (VR) environments are calculated and projected within the computer, we include video games, metaverses, simulators, etc. in this category. Mixed reality (MR) refers to Augmented Reality environments created by the synchronization and simulation of virtual objects that are adapted to the real world. Conversely, Augmented Virtuality Environments (AV) synchronize the presence of real-world objects in the virtual world. For example, this includes Location Based Entertainment (LBE), which leverages different forms of synchronizing the real world within the virtual world. LBE incorporates captured movement and modelling of physical space in synchronization with objects, people and visual textures. The third category in our typology is Ubiquitous Environments, which include physical computing, smart clothes and the Internet of Things. In this case, usage becomes the prime focus, the interface becomes invisible, and the object integrates into the real-world environment using various forms of geolocation in relation to the individual. For their part, Recorded Environments are based on $360^{\circ}$ live surround capturing and recording of action in video format and point clouds. To avoid confusion, it is important to note that 
Recorded Environments cover all forms of data capturing, from $360^{\circ}$ video to Lidar technologies and up to photogrammetry and videometry [18]. The final category is Projected Environments (outside of computers), which include CAVE / FullDomes / Multi-screen and video mapping. These projected environments are created by synchronizing multiple projectors. This is referred to as Spatial Augmented Reality (SAR) [19]. In other words, a building, dome, sculpture or any other type of medium is augmented using various technologies and video projections. All of these environments help structure the narrative dimensions of immersive storytelling. The principal hypothesis of this article is that the World Building framework can contribute to research-creation in immersive storytelling.

\subsection{World Building}

The World Building approach upholds an industrial practice of transmedia storytelling that makes it possible to provide franchises, television shows, films and video games with a degree of unity with respect to the narrative, aesthetics and formal aspects, for distribution across a wide array of platforms [20]. However, in light of an examination of the books written on this topic, World Building can serve as a model for many diverse practices, from literary practice and futuristic exploration of architectural scenarios to the production of expanded reality (or cross reality) (XR) projects.

To properly circumscribe the World Building approach, it is worth relating a very telling anecdote. During a conference entitled "World Building the Marvel Way," Bill Rosemann, Director of Marvel Games, told the story of a Captain America fan who, during the 2003 Boston Marathon, decided to turn back to help out the rescuers; Roseman then precisely detailed his vision of the term: "When Marvel does it job right, we all do our job right, we create fictional world that can then impact the real world" [21]. Although there are many ideological elements to take into account in the context of such an assertion, it does highlight the foundations of the World Building conceptual framework, i.e., the complex relationship between the primary world (real world) and the secondary world (imaginary world). The imaginary world exists in parallel with the real world. Similarly, as Bielskyte noted in a critique of traditional media, "We have to see the world, not something that we see in a frame, but really as a world that would actually exist as parallel world to our physical world" [22].

In a similar vein, according to Mark J. P. Wolf, World Building consists in designing secondary worlds inspired by the real world and which establish the underlying conditions that enable stories to emerge. Wolf uses the term "subcreation," which he himself borrowed from J. R. R. Tolkien [23], and notes that the "world" can be imagined in a geographical as well as in an experiential form. A world can contain anything that can be experienced by characters within its fictional realm, and the same goes for the events that can give direction to their lives. Interestingly, Wolf makes reference to the etymological meaning of weorld, an old German term meaning "all that concerns humans" [24].

Wolf also describes World Building as being organized by three broad rules of design: invention, completeness, and consistency [25]. The first rule is invention. Invention grows out of, but also distances itself from, the real world by creating new hybrid figures in the design of technologies, cultures, artifacts, institutions and new forms of ecosystems. In our view, Bielskyte gives a fairly good description of the relationship that the principle of invention establishes between the secondary and primary worlds. To help bring this fictional world into being, Bielskyte asks a series of questions: "[...] what are the landscapes we do not know? What other plants, what are the animals. What is the contemporary art? What are 
the sort of scientific breaktroughs or scientific locations? And sort of really looking at the richness of diversity, plurality of our world and our culture and the environment that we live in [...] looking at it, at the shapes of theses colors, of theses stories and theses gestures and transforming them. Taking the best of reality, remix in it in a new way, changing the scale of things and adding a layer of magic and imagination" [26].

The second rule, completeness, has to do with the internal limits, or boundaries, that the world sets up. In other words, it refers to the quantity of information available to the viewer, visitor or user exploring the world. The idea is to enable this individual to find and verify the information they need to guide their actions. In other words, the question to ask is whether the explanations provided by the established systems inform the individual of the rules that organize the world, while also keeping its boundaries concealed. How can the world be contained, both in terms of territory and imagination? What strategies are employed to bound the world's geography while keeping its borders invisible to the world's "guests"? These two components must support the third rule, which is consistency. In other words, invention and completeness obey fictional rules which must, in the context of the subcreation, give the world a high degree of internal plausibility. Importantly, plausibility does not reside in the world's actual relationship to the real world, but rather in the world's consistency via the functioning of rules and fictional characters. Completeness refers to the degree of consistency structuring the world's ecosystem. A more complex world will make completeness progressively more complex. As such, the details are just as important as the whole. Indeed, the details must be in keeping with the general structure of the pre-established world. It is therefore important to meticulously follow the general structure of the system of rules that were put in place. Going along with imaginary systems, inventions and rules requires a temporary suspension of disbelief. For example, in the recent TV series "West World" (2016), the participants in a Far West simulation can break many actual laws of the real world. We accept the actions of the characters because we know that this fictional world is populated with androids. Consistency dictates even the smallest details, such as clothing texture, weapon design, and the use of certain transportation modes. All these elements must, by their presence, reinforce the logical and formal unity of the constructed world. It is thus no coincidence if many of these worlds are geographically located on islands or spaceships. The spatial confinement of the players in the story helps mark out the realm of action; indeed, isolation justifies the world's boundaries but also its social structures, architecture, costumes, etc. The three rules of World Building thus allow for devising an imaginary world that abides by its own internal rules and boundaries.

In 2008, Alex McDowell, Director of the World Building Institute (WBI) at the University of South California Department of Cinematic Arts, summed up the approach as follows: "World Building designates a narrative practice in which the design of a world precedes the telling of a story; the richly detailed world becomes a container for narrative, producing stories that emerge logically and organically from its well-designed core" [27]. Given this statement, it should come as no surprise that McDowell was Production Designer for the film Minority Report (2002). When it came out, the film foreshadowed the future use of dematerialized screens and multitouch technology. According to Wolf and McDowell, this approach makes it possible to generate stories in accordance with a transauthorial and transmedial logic [28]. Building fictional worlds also provides direction for urban planners and architecture designers. In an article entitled "World Building and the Future of Media. A case Study - Makoko 2036," Laura Cechanowicz, Brian Cantrell and Alex McDowell relate an experiment conducted by a hodgepodge group of students, architects, interactive media designers, musicians, engineers, urban planners, animators, directors and artists. Drawing inspiration from a specific location and culture, specifically the Lagos Lagoon in Makoko, 
Nigeria, the group explored the articulation of different fictional scenarios, from both a story and technology standpoint. In this interdisciplinary World Building context, the authors of this last article wrote, "Any storyteller can weave a compelling narrative, but world builders create storyworlds that support myriad stories by multiple storytellers across disparate platforms - including those platforms that may not yet be named" [29]. It thus appears that the world exists in itself, outside of any sort of media embodiment, and thus serves as a basis for the infinite generation or proliferation of stories and substories. Whether transdisciplinary, transmedia or transauthor, any form of subcreation constitutes a world that guides the expressive use of digital technologies. Clearly, given its all-encompassing nature, beyond its commercial and industrial applications, the World Building approach is particularly fertile ground for designing immersive experiences. Without getting into details, Wolf distinguishes between three perceptual states: immersion, absorption and saturation. It can be postulated that any current or future world, whether existing or yet to be designed, can provide the basis for building a world obeying specific perceptual states. The visitor will thus be plunged into the world (immersion) and captivated by the elements that make up the storyworld (absorption). The world's complexity makes it impossible for the individual to experience all of its elements (saturation). Outside of any particular narrative structure, such as a story arc, being immersed in a fictional creation as a witness or participant brings into play the prime characteristic of digital storytelling, which is interactivity. A world existing and functioning according to its own specific organizational rules allows the person immersed in it to experience the fictional world according to their own point of view. It is worth reiterating that the world determines the preconditions for the emergence of multiple stories. Like a virtual world persisting in the cloud of digital data and evolving according to its own unique logic, by existing as a fiction, a world, an environment, a culture, etc., World Building is an immersive construct. On this topic McDowell, who primarily expresses this idea in talks and presentations, mentioned in a radio interview: "I think VR is just an opportunity to think about a completely different medium. Once you take that step and you get technology that allows you to immerse yourself completely in an unfamiliar environment, my sense is that this environment will provoke the stories that it requires" [30]. Hence, the environment engenders its own narrativity, by the mere fact of its existence. What is at play is therefore no longer a narrative structure, but an invented world that exists within certain boundaries and is organized according to its own specific rules.

From a practice perspective, there are many design rules and points of entry when producing a (story)world. For example, one can use the language of the inhabitants or the geography of the story as a starting point. Moreover, it is essential to determine the historical period (be it real or fictional), the planet, the city, architecture, the building, the institutions, etc., just as it is necessary to determine the characters' emotional characteristics, interaction rules, personal histories, powers, and so forth. Indeed, it is important for all these elements to be determined since they lay the groundwork for viewers to experience a degree of expectation within the context of the story. There is no specific methodology or recipe for designing a "storyworld"; rather, an infinite number of points of entry can be considered. The same is true of games, i.e., there are no specific rules in designing a storygame [31]. However, among all the available choices (characters, languages, cultures, institutions, etc.), one design variable is paramount: spatiality, which includes geography, architecture, location and space. In other words, spatiality, whether imagined in a literary or virtual form, is at the core of the practice of World Building. In fact, the practice of environmental storytelling is based precisely upon this affirmation. 


\subsection{Environmental storytelling}

Inspired by the storytelling strategies used in the architectural design of theme park attractions, the field of environmental storytelling is premised on setting up the conditions for stories to emerge by first constructing the space. In the words of Don Carson, former theme park experience designer, "One of the trade secrets behind the design of entertaining themed environments is that the story element is infused into the physical space a guest walks or rides through" [32]. In this context, when it comes to both real-world and virtual environments, the practice comes down to articulation strategies. Carson also discusses the conditions for dialogue between real-world and virtual spaces. Based on an analysis of his design experience, he defines how the specific narrative structure of theme parks' architectural design helps better understand the field of environmental storytelling. Along similar lines, Henry Jenkins mentions in his text "Game Design as a Narrative Architecture" that spatiality is central to designing games, whether tabletop or virtual: "Game designers don't simply tell stories; they design worlds and sculpt spaces" [33]. Jenkins goes a step further by maintaining that the field of "environmental storytelling creates the preconditions for an immersive narrative experience in at least one of four ways: spatial stories can evoke pre-existing narrative associations; they can provide a staging ground where narrative events are enacted; they may embed narrative information within their mise-en-scene, or they provide resources for emergent narratives" [34]. Hence, the imbrication of places, be they open or closed, affords the interacting player or actor some agency enabling them to create their own narrativity. On the website "Gamasutra," Bart Stewart states that "Environmental storytelling is the art of arranging a careful selection of the objects available in a game world so that they suggest a story to the player who sees them," adding that, "Environmental storytelling is less direct. Instead of explicitly describing events, environmental storytelling shows the final outcome of a sequence of events, then it invites players to make up their own stories about what happened to cause that outcome" [35]. This goes to show that the player's agency conditions their narrative experience of the space. On this topic, Mary Laure Ryan distinguishes between different relationships to space from a strategic and emotional point of view. The space is emotional in that "the experience of that space is associated with emotional values," and Ryan goes on to say, "Whereas the emotional design of the space constructs the subject by the relationship he or she has with their ambient environment, strategic design constructs the subject as possessing agency" [36]. Hence, storytelling is also based on space as perceived from an aesthetic as well as functional standpoint. This means it is important to determine whether the subject will recognize a familiar (emotionally resonant) environment or a strategic one that serves functional goals. More specifally, the strategic space can be in the form of a sandbox, i.e., it can be viewed as pure indeterminacy, or in the form of level design that responds to the strategic requirement of a particular affordance of space according to the construction of the player's experience.

\section{World Building: Full Dome research-creation projects}

The World Building hypothesis emerged progressively and spontaneously as an inescapable solution in the context of creating a number of research-creation projects in the Full Dome format. First, the Environmental Storytelling hypothesis structured the scripting of the projects Re-Génération (2015) and Les Planètes (2018). The Illumination Frankenstein (2018) project was guided by the World Building hypothesis throughout, from ideation all the way up to performance in November of 2018 [37]. 


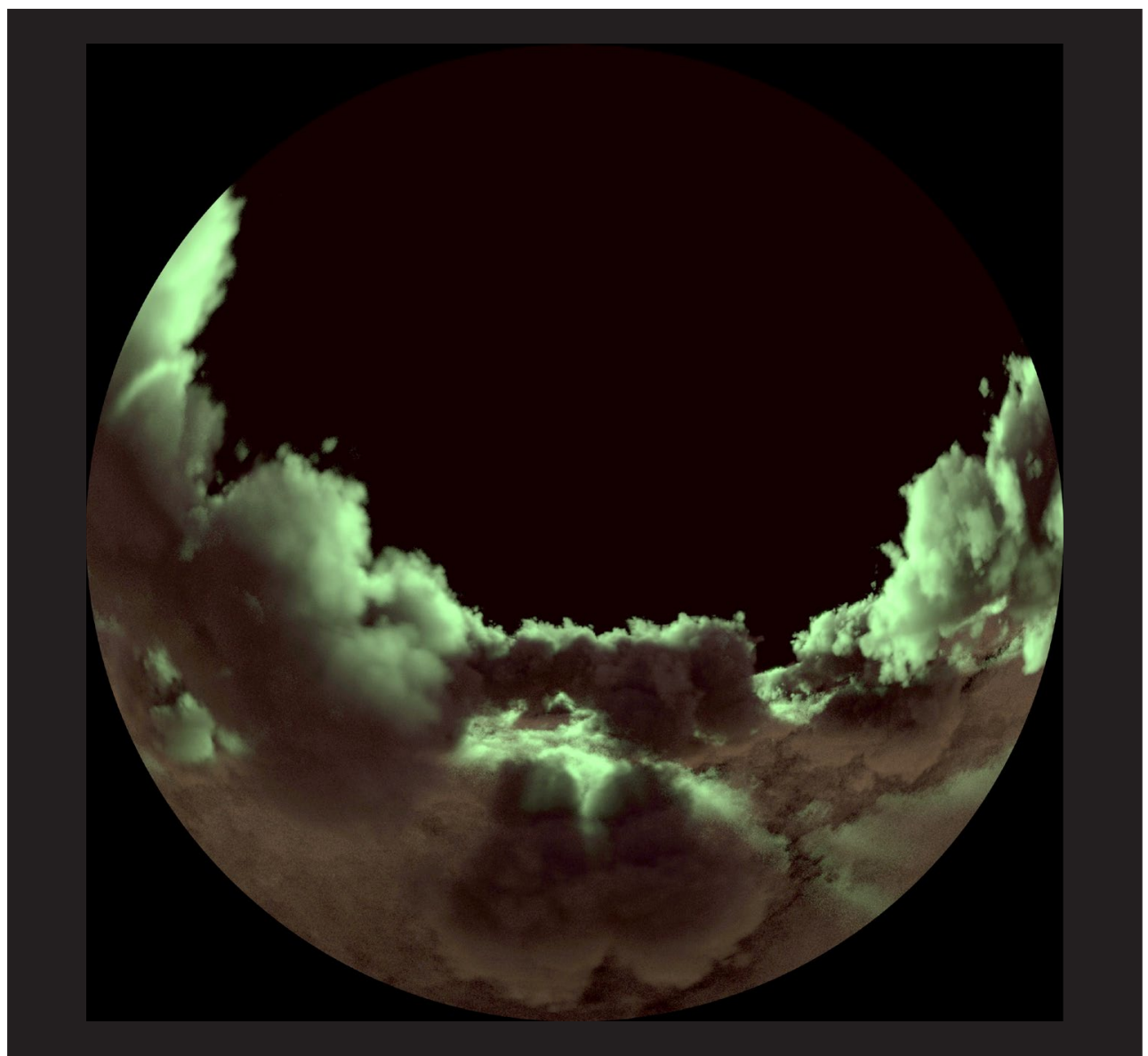

Fig. 1. Re-Génération. Société des Arts Technologiques. Cité des sciences et de l'industrie (2015)

To properly understand the evolution of the hypothesis of using the World Building theoretical framework, it is worth providing an overview of key moments in this evolution. In 2008, as part of its cultural activities on the heels of Cop21 in Paris, the Société des Arts technologiques was commissioned with creating an immersive work on the theme of climate change. David McConville, then Director of the Buckminster Fuller Institute, responded by imagining a new form of transmission based on relationships between the human body and the environment via embodiment approaches. His idea was to use environmental visualizations from NASA, simulations of living organisms and synthetic environments to convey a certain vision of climate change through the exploration of the sensory dimensions of the immersive medium that was put in the service of the narrative. The intent was to arrange multiple metaphors in order to create a sensory experience demonstrating that human beings are not separate or isolated from the ecosystems they are changing and transforming. The project, entitled Re-Génération, observed the natural world from the standpoint of the time sequence of day and night, the tides, and land movements, all viewed as one giant breath extending across the globe [38]. 
The narrative scenario, inspired by the Gaia hypothesis, consisted in identifying different levels of knowledge emerging from studies on the broad theme of regeneration. The second level involved writing a narrative presented in the form of a meditation on climate. The story behind the project, written by David McConville, was scripted on the basis of a succession of metaphors drawing links between the balance of ecosystems and the human body. The narration, or storytelling, was thus built on a succession of continually shifting environments. The structure was guided by the observation that narrativity, in a Full Dome situation, no longer plays out through images but through the environment. Once conceptualized, each environment in this scenario was produced by several teams composed of artists and directors. It is important to point out that while it was performed in the immersive format of a Full Dome projection, the project was created using a transauthor approach.

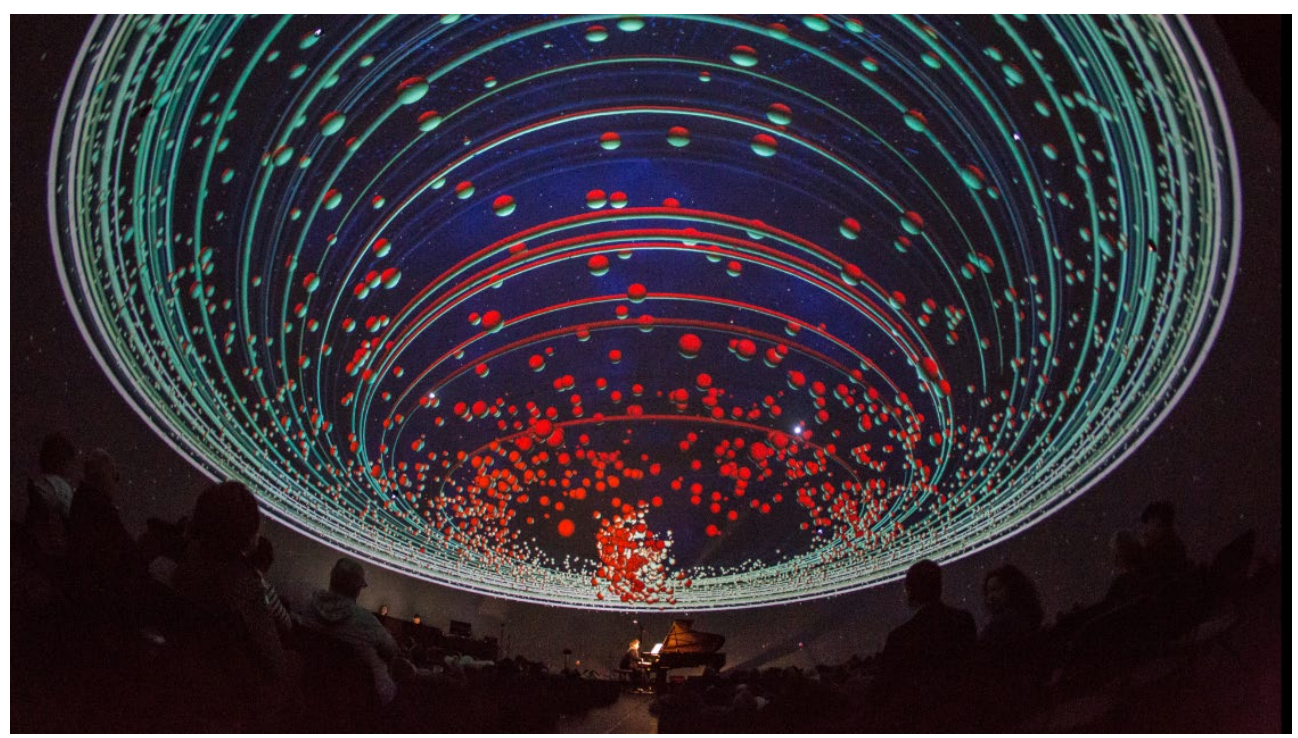

Fig. 2. Les Planètes. Société des Arts Technologiques (2018). Photo: Nathanael Corre

The second experience where the environmental storytelling conceptual framework was used was entitled Les Planetes (2018) [39]. Globally speaking, this project consisted in a series of environments that responded to music and that could, in keeping with a Transmedia aesthetic, be transposed to single-screen, multi-screen, panoramic, virtual-reality and Full Dome formats. The first version of the project was presented as an immersive Full Dome concert generated entirely in real time. The project involved creating a light-piano that entertained a visual dialogue with pianist Louise Bessette regarding a new interpretation of Les Planètes by composer Walter Boudreau. The light-piano enabled the projection of dynamic constellations of small spheres scattered across the dome's projection space. The visual shapes were based on analysis of different sections of the musical score. Each section was considered an abstract subworld, as it was divided into as many planets as exist in our solar system. Using environmental storytelling directly resulted in generating the immersive experience through a series of interactional rules between virtual objects. Thus, each environment explored an array of music and sound visualization strategies, and the succession of visualizations yielded a form of abstract storytelling or sensory storytelling. In this context, environments of abstract shapes constituted spaces of mental projection (similar to huge Rorsach ink blots) in which viewers drew their own meaning from the aesthetic 
shapes as they allowed their attention to wander. Along the same lines, this approach fundamentally relying on transmedia had already been explored in two previous projects carried out in collaboration with the composer Alain Thibault, namely White Box (2011) and Enigma (2017-2019) [40].

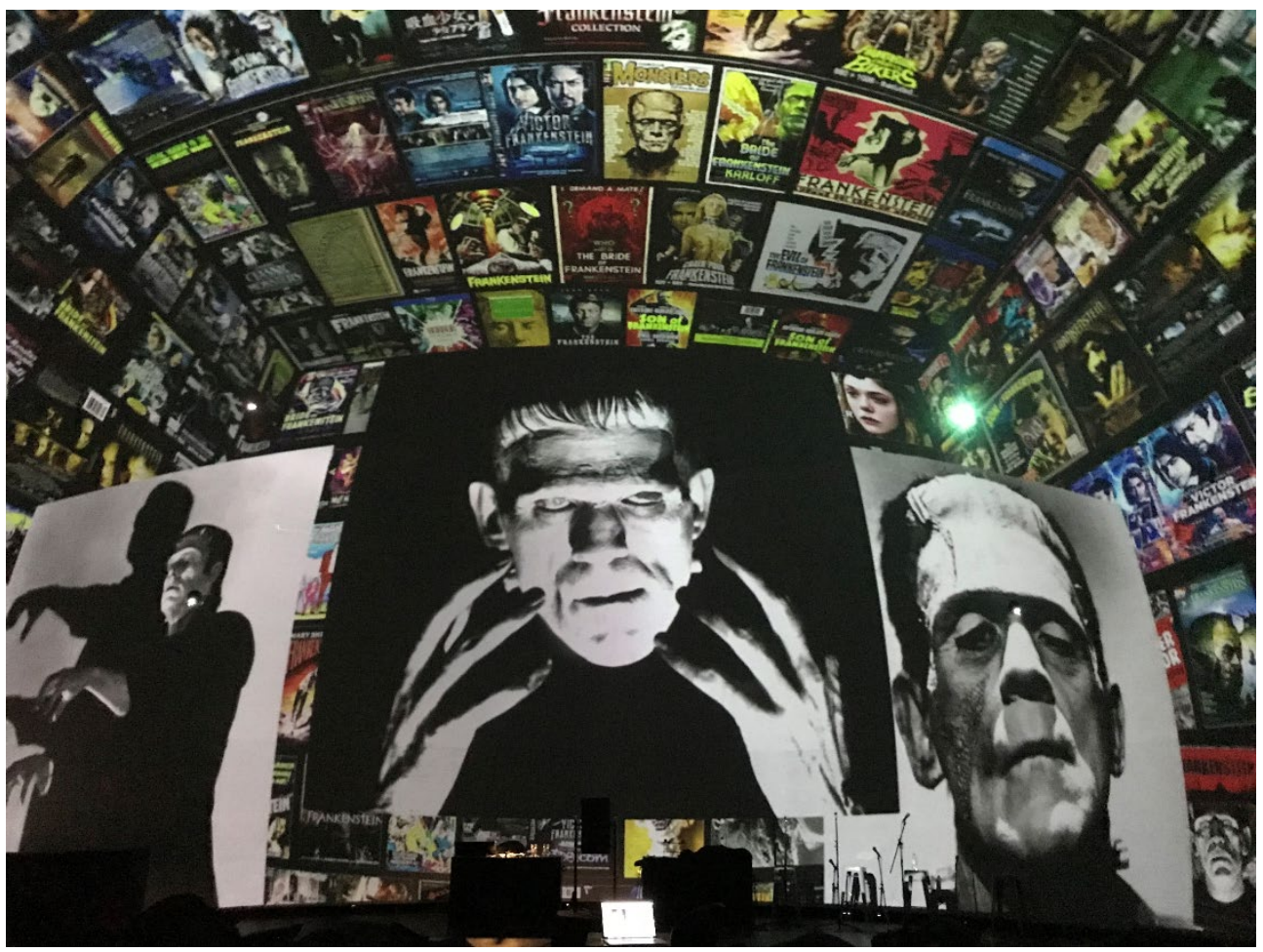

Fig. 3. Illumination Frankenstein. Société des Arts Technologiques (2018). Photo Yan Breuleux

The third Full Dome World Building experience that used environmental storytelling was based on the fictional world of Frankenstein as imagined by Marie Shelley, whose novel came to popularity in movie theatres. The project consisted in an immersive lecture by sociologist Céline Lafontaine [41]. In this case, we adopted the opposite approach by presenting the elements which, in the actual romantic era, shaped the world of Dr. Frankenstein and his creature. Thus, Illumination Frankenstein takes the shape of a spatialized story to present a succession of environments composed of archives from the complex world that gave rise to Shelley's scientific vision. This brings up the question: What was the real world that inspired the novel? For example, a study of the novel reveals that Frankenstein was put together from human and animal corpses, and was vegetarian. An interpretation using a World Building approach could allow for a new modelling of the creature, using details provided by the author. Indeed, studying the novel and comparing its different versions would allow for composing a coherent virtual world in keeping with a World Building logic, if we examine the story in its minute details. The immersive lecture helped expose the actual world that give birth to the novel Frankenstein. Following a narrative structure of environmental storytelling, composed as a journey within a myriad of immersive environments, the project was, fundamentally, underpinned by an understanding of different ways of applying the World Building model. The idea was also to show how, "Two hundred years after its literary birth, the prescient light that this prophetic novel shed 
on the beginnings of modernity still shines today and invites a reflection on the conquest, manipulation and devastation of the living world" [42]. In other words, by its very structure, the project attempted to draw links between the primary and secondary worlds of Frankenstein, which are at once romantic and modern.

\subsection{World building: virtual reality research-creation projects}

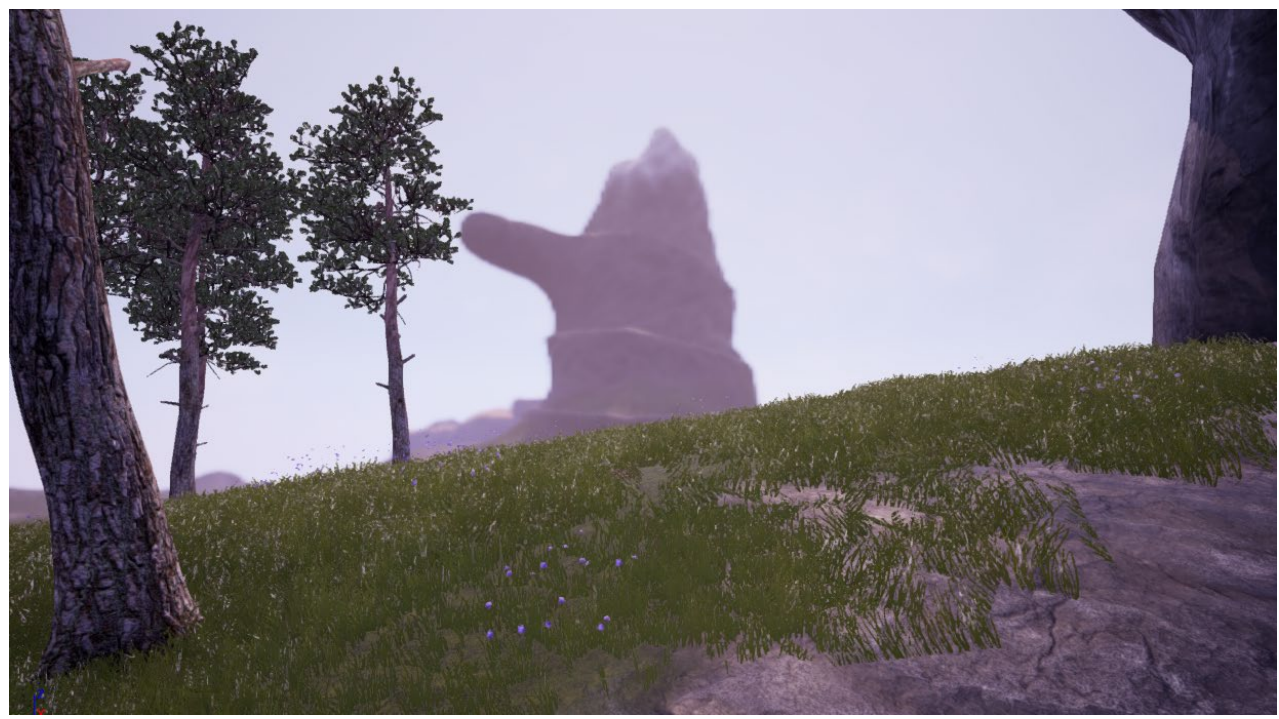

Fig. 4. PeterPan $360^{\circ}$, Bruno de Coninck, NAD - UQAC (2019).

The theoretical framework of World Building has helped guide the research and structure the practice of a number of students at the NAD school pursuing research-creation in the course of their master's studies (Master 2). Bruno de Coninck has used different media to model a virtual environment of the world of Peter Pan by blending a number of different interpretations of this world imagined by James Matthew Barrie in the early 20th century $(1904,1911)$. Once the prototype was completed, it gave rise to a series of hypotheses for transcoding the world in different formats. The last version consisted in generating a virtualreality world. To achieve this, Coninck compared different descriptions of this world and then modelled a 3D narrative environment that served as a medium for various game mechanics. Semantic blending of countless interpretations of the tale were used to add to, complement and compose a complete virtual environment. 


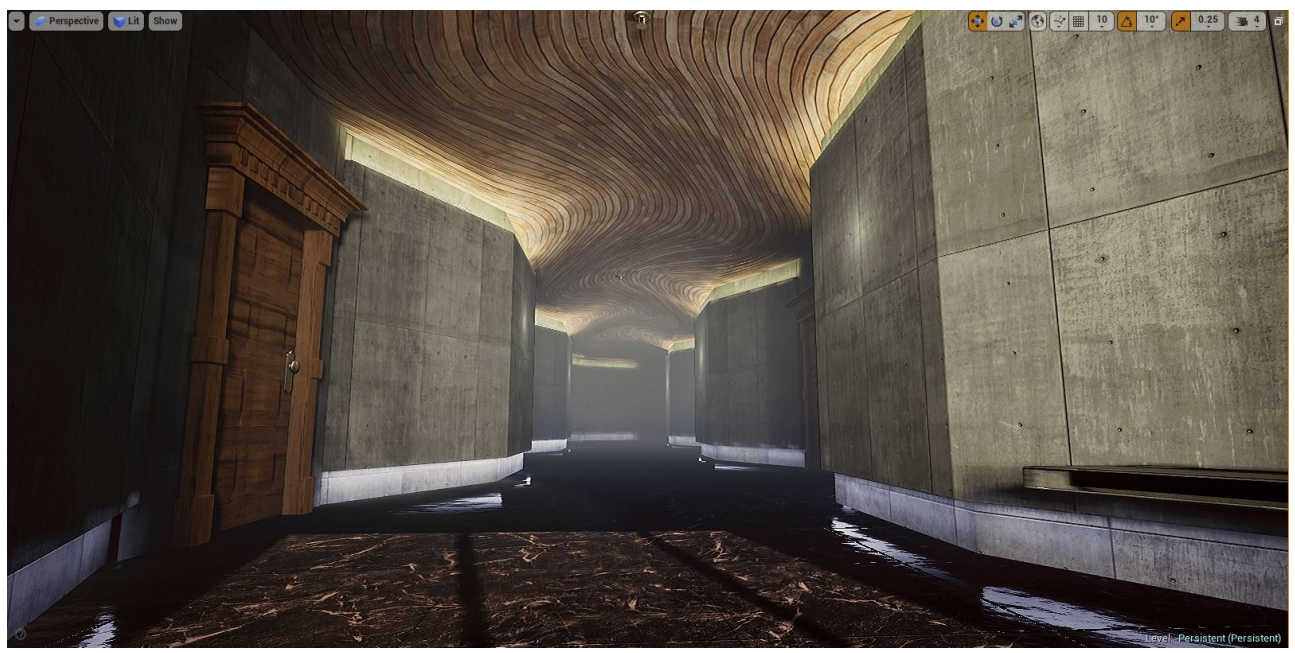

Fig. 5. Hybrid environment. Simon Therrien (2019).

For his part, Simon Therrien has imagined a hybrid world based on a cross between the world of Franz Kafka's novel Métamorphose and Jim Henson's Labyrinth. His environmental storytelling project has taken the shape of designing a new video game depicting a number of fantastic environments as settings for action. Therrien's research has primarily involved defining a series of conceptual tools to nourish reflection on practice.

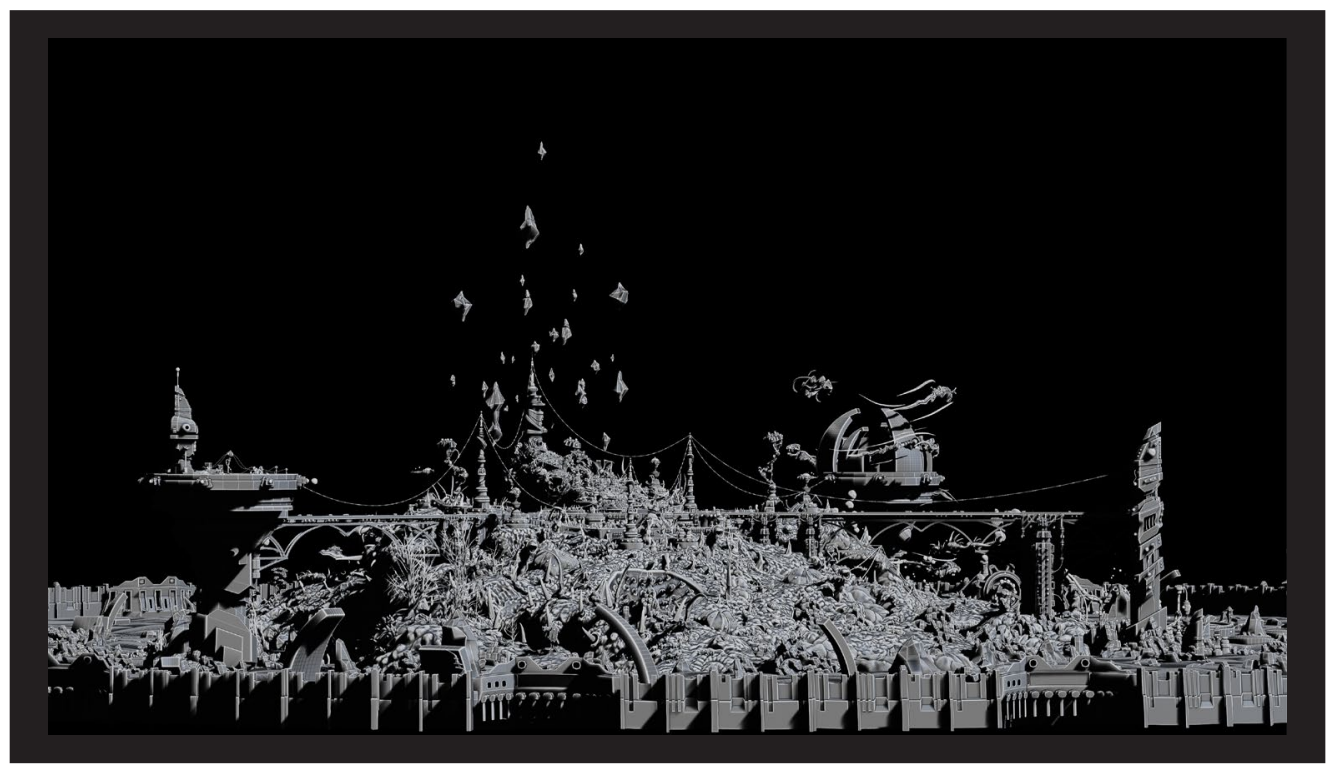

Fig. 6. Cités-Mondes. Samuel David (2019).

Samuel David, with his project Cités-Mondes, has worked on environmental storytelling by observing the creative dimensions of 3D modelling while adopting a reflexive practitioner approach. Thus, David has created an animated universe determined by fictional 
rules for creating animated ecosystems. In reality, he modelled a complete world that functions according to its own internal rules. Objects conceived in this way could enable writers to imagine stories, intrigues, tensions, etc. This is because the environment serves as the medium for an imaginary narrative space. In a different vein, Eric Filion (also known by the pseudonym $\mathrm{Vj}$ Nokami) has drawn inspiration from the study of the specific environment of Iceland to make an immersive film on the unique sense of Solastagia described by Glen Albrecht [43]. The goal of Filion's project was to help the viewer visualize this unique emotion, characterized as a sort of anguish provoked by the gradual shifting and transformation of our natural environment.

\section{Conclusion}

The theoretical framework of World Building offers a springboard for analyzing the practice of environmental storytelling in the field of Spatial Augmented Reality (SAR), and more specifically in the Full Dome format with its succession of environments. In sum, our analysis of projects upholds that the World Building approach structures the creation of storyworlds and storygames that can be adapted to multiple apparatuses and installations. As such, and viewed from an art and design standpoint, this approach makes it possible to structure storytelling projects while taking into account a diversity of projection platforms. To illustrate this assertion, one could mention for example the fact that the overarching theme of a museum exhibit can constitute a storyworld. In this context, the different artifacts are produced using a transmedia approach. After conducting our analysis, we have also noted that the world's organization is based on collaboration between players in the academic and institutional milieus, and with professionals from multiple disciplinary backgrounds. In our view, the transauthor dimension is especially relevant as it helps structure the contributions of all the players involved. Another interesting fact is that environmental storytelling can be embodied both in the exhibit's scenography and in the media design of parallel worlds, virtual reality experiences, etc. In this context, the experiential design approach adapts particularly well to the storyworld formulated by all of the players, especially since all historical periods obey rules pertaining to story structure and construction. Hence, the characteristics of World Building, as formulated by Wolf, provide a theoretical framework for organizing the relationship between art, design, technology and storytelling, by following a certain logic. Whether in museology, documentaries, films or digital art projects, this relationship helps define a new form of transmedia storytelling. In all cases, the process is based on a series of critical steps. The first is to immerse oneself in the historical world, and the second, to imagine a map, a place, an environment. However, the elements entailed by each step vary depending on the project; depending on the case, one may need to include interactions with the characters, the era, technologies, and common objects. From this world, this subcreation, all media choices become possible.

In our case, in the context of our recent research and that of our students, the worlds have, globally speaking, been based on the use of an existing story. The underlying research makes it possible to imagine a certain historical environment and to convey knowledge through a given storyworld, thus giving visibility to the paradigm on which the knowledge is based. In this general context, the theoretical framework of World Building also transforms the paradigm from which the project is created. The story plays out through the environment in which the action occurs, but partakes in what Bielskyte describes as a storyworld experience. The storyworld experience is developed in the field of VR films via close collaboration between four key individuals: the director, the production manager, the engineer (or technical artist) and the interaction designer. Each has a specific role to play: 
1- the director is in charge of the story and the emotional design, 2- the production manager carries out environment-related tasks, whether in terms of architecture or narrative, 3- the engineer fleshes out how the story will be conveyed, and 4- the interaction designer determines mechanics and logics of interacting with the world. This collaboration somewhat diminishes the director's status as a demiurge. As the designer of the experience, the director adapts to and collaborates with an existing world and the other team members. The engineer now participates in storytelling, and the interaction designer (as a specialist in relationships with the world) determines the strategies the user will use to interact with the world. This said, it is important to understand that this structure brings into play not just skills, but also a space of horizontal communication. Indeed, each player in the process observes the issues surrounding the story, the environments, the interaction and the technological means, all from the characteristic angle of their particular profession. Thus, in a World Building approach, each role involves exploring the conditions for bringing the story and environment into existence (from both a modelling and technological standpoint) by connecting them with different forms of interaction.

The project Dans la lune (2019), currently being developed under the direction of Marc Caro in collaboration with a group of students and teachers from the École Supérieure d'architecture de Nantes, consists in a VR film experience inspired by a hybridization of the world of the film magician Georges Méliès and the retrofuturistic aesthetics of Jules Verne, whose Machines de Nantes are an outstanding example of this aesthetic [44]. The construction of the film's immersive décor is a central part of articulating the experience. Consistent with the World Building framework, constructing this film involves the combination of a world and the building of a storytelling environment where the action will take place. In this context, the theoretical framework of World Building serves as a foundation to analyze the project's narrative dimensions as well as its creation process.

In light of current trends in immersive technologies, it appears evident that the transmedia premise of storyworlds allows for adapting stories across different forms of technological environments. One avenue to explore would be to look at how the model applies to the four worlds of storytelling as set forth by Scott A. Lukas in 2013 [45], which was linked to the theory of possible worlds as studied by Wolf and Ryan. [46], [47]

According to Lukas, the primary world is the realm of reality (a), the second is imaginary (b), the third is experiential (c) and the fourth is the world of guests (d). These four perspectives constitute as many trades and complementary endeavours and approaches. The first world consists in the materiality of built structures, from an urban planning, architectural and industrial standpoint. The second world, that of the imagination, stems from literature, fiction and all forms of visualization (maps, models, patterns, language, etc.). The third world, experiential, comprises technological environments such as those described in this article, and the design of visitor interactions. The fourth world, or visitor world, corresponds to the perspective of the receiver. Clearly, our field is located in the humanities and social sciences. How can the unique perspective of the individual be understood in the context of the interrelationships between all these worlds? Moreover, what knowledge would help to include guests in the storytelling process? In our case, it is clear that we are in an interdisciplinary context. The next step of the present study would be to establish a design methodology making it possible to create a narrative continuum between these different worlds, beyond their technologies. This brings into play the hypothesis of World Building as a continuum of mixed reality or cross-reality—not technological, but rather narrative and experiential, in nature. 


\section{References}

1. Miller, Carolyn Handler. Digital storytelling: A creator's guide to interactive entertainment (Focal Press, 2014), p.14.

2. Patent of the Telesphere Mask invention:

[https://patents.google.com/patent/US2955156A/en], page accessed on February 6, 2019.

3. Sutherland, Ivan E. "The ultimate display." Multimedia: From Wagner to virtual reality (1965): 506-508.

4. A detailed chronology is available in chapter 1 of the following book: Alexander, Bryan. The New Digital Storytelling: Creating Narratives with New Media--Revised and Updated Edition. (Abc-clio, 2017).

5. Murray, Janet Horowitz. Hamlet on the Holodeck: The Future of Narrative in Cyberspace (New York: Free Press, 1997).

6. Balet, Olivier, and Gérard Subsol, Torguet, Patrice. Virtual Storytelling; Using Virtual Reality Technologies for Storytelling: International Conference, ICVS 2001, Avignon, France, September 27-28, 2001, Proceedings. Vol. 1. (Springer, 2001). P.2.

7. International Conference for Interactive Digital Storytelling : [https://icids2018.scss.tcd.ie/], page accessed on February 6, 2019.

8. New York Times: [https://www.nytimes.com/2019/01/25/theater/hamlet-virtualreality-google.html], page accessed on February 6, 2019.

9. Future of Storytelling: [https://futureofstorytelling.org/], page accessed on February 6, 2019.

10. MIT Open Documentary Lab: [http://opendoclab.mit.edu/], page accessed on February 6, 2019.

11. Ropert-Dupont, Émilie. Journalisme et réalité virtuelle: Émotion ou information?. (Éditions L'Harmattan, 2017).

12. Centre PHI: [https://phi-centre.com/en/event/fost-2015-en/], page accessed on February 6, 2019.

13. The new storytellers: [https://phi-centre.com/en/event/the-new-storytellers-5-en/]

14. Douglas Trumbull : [https://www.youtube.com/watch?v=47wO-Az22v0], page accessed on February 6, 2019.

15. Gödde, Michael, Frank Gabler, Dirk Siegmund, and Andreas Braun. "Cinematic Narration in VR-Rethinking Film Conventions for 360 Degrees." In International Conference on Virtual, Augmented and Mixed Reality, pp. 184-201. Springer, Cham, 2018. See: Tricart, Celine. Virtual Reality Filmmaking: Techniques \& Best Practices for VR Filmmakers. Taylor \& Francis, 2017. Also worth mentioning is: Bucher, John. Storytelling for virtual reality: methods and principles for crafting immersive narratives. Focal Press, 2017.

16. Milgram, P., Takemura, H., Utsumi, A., \& Kishino, F. (1995, December). Augmented reality: A class of displays on the reality-virtuality continuum. In Telemanipulator and telepresence technologies (Vol. 2351, pp. 282-293). International Society for Optics and Photonics.).

17. The New Zealand VR/AR Association (NZVRARA). Virtual gets real. The Explosion of Cross Reality in New Zealand, report one, September 2017.

18. Breuleux, Y. Narrativité immersive pour Vr et FullDome. Art \& Technologie. Effects Mtl, 2017. Palais des congrès de Montréal. 
19. Bimber, Oliver, and Ramesh Raskar. Spatial augmented reality: merging real and virtual worlds. AK Peters/CRC Press, 2005.

20. Boni, Marta, ed. World Building: Transmedia, Fans, Industries. Amsterdam University Press, 2017.

21. World Building the Marvel Way, Bill Rosemann, Marvel Games (2017):[ https://youtu.be/p3J2ZuOmFWw?t=120 ], pages consulted on February 6, 2019. Also worth mentioning is an article on the links between the Marvel empire and the World Building approach: Jeffries, Dru. " The Worlds Align." World Building Transmedia, Fans, Industries (2017): 287.

22. Monika Bielskyte, The New Storytellers II: World-building: Best Practices for Creating Story Worlds: [ https://youtu.be/jtr0AZK64Do?t=1093], page accessed on February 6, 2019.

23. Wolf, Mark JP. Building imaginary worlds: The theory and history of subcreation. Routledge, 2014. P.19.

24. Wolf, Mark JP. Building imaginary worlds: The theory and history of subcreation. Routledge, 2014. P.39.

25. Wolf, Mark JP. Building imaginary worlds: The theory and history of subcreation. Routledge, 2014. P.44.

26. Monika Bielskyte, The New Storytellers II: World-building: Best Practices for Creating Story Worlds: see (24:42): [https://youtu.be/jtr0AZK64Do?t=1093], page accessed on February 6, 2019.

27. The World Building Institute: [http://worldbuilding.institute/about], page accessed on February 6, 2019.

28. Wolf, Mark JP. Building imaginary worlds: The theory and history of subcreation. Routledge, 2014. P.28.

29. Cechanowicz, Laura, Brian Cantrell, and Alex McDowell. "World Building and the Future of Media: A Case Study-Makoko 2036." IEEE Technology and Society Magazine 35, no. 4 (2016): 28-38.

30. Voices of VR: Alex McDowell on World Building in Storytelling, [voicesofvr.com, podcast 309] (2016), page accessed on February 6, 2019.

31. Montfort, Nick. "Interactive Fiction as

'Story, ' Game, 'Storygame, 'Novel, ' World, '’Literature, 'Puzzle, 'Problem, ' Riddle,' and 'Machine. '." First Person: New Media as Story, Game, and Performance (2004).

32. Don Carson:

[https://www.gamasutra.com/view/feature/131594/environmental_storytelling_.php], page accessed on February 6, 2019.

33. Jenkins, Henry. "Game design as narrative." Computer 44, no. 53 (2004): 118-130.

34. Jenkins, Henry. "Game design as narrative." Computer 44, no. 53 (2004): 118-130.

35. Bart Stewart, Gamasutra:

[https://www.gamasutra.com/blogs/BartStewart/20151112/259159/Environmental_Stor ytelling.php], page accessed on February 6, 2019.

36. Ryan, Marie-Laure. "L'expérience de l'espace dans les jeux vidéo et les récits numériques." Cahiers de Narratologie. Analyse et théorie narratives 27 (2014). P.2.

37. Re-Génération: [http://www.uqac.ca/portfolio/yanbreuleux/recherche-creation/], page accessed on February 6, 2019.

38. See: [http://sat.qc.ca/cop21], page accessed on February 20, 2019. 
39. See: [http://sat.qc.ca/fr/planetes], page accessed on February 20, 2019.

40. See: [https://vimeo.com/purform/enigma], page accessed on February 20, 2019.

41. See: [https://socio.umontreal.ca/repertoire-departement/vue/lafontaine-celine/], page accessed on February 20, 2019.

42. Illumination Frankenstein: [https://sat.qc.ca/fr/evenements/illumination-frankenstein], page accessed on February 20, 2019.

43. The theses of Bruno de Koninck, Simon Therrien, Samuel David and Eric Filion will be made available during the summer of 2019 on the following website:

[https://constellation.uqac.ca/], page accessed on February 20, 2019.

44. The project is currently under production:

[https://www.instagram.com/architecture.representation/],

[https://www.instagram.com/architecture.representation/] and

[https://architecturerepres.wixsite.com/decor360], page accessed on February 20, 2019.

45. Lukas, Scott. The immersive worlds handbook: designing theme parks and consumer spaces. Focal Press, 2012. P.230.

46. Bell, Alice, and Marie-Laure Ryan, eds. Possible Worlds Theory and Contemporary Narratology. University of Nebraska Press, 2019.

47. Wolf, Mark JP. Building imaginary worlds: The theory and history of subcreation. Routledge, 2014. P.17. 